\title{
The Characteristics and Effects of Fan Community Communication
}

\section{——Take the Series 227 Events of Zhan Xiao's Fan as an Example}

\author{
Jingyi Chen ${ }^{1, *}$ \\ ${ }^{1}$ Changsha University of Science \& Technology, Zhangzhou, Fujian, China \\ *Corresponding author:1131595214@qq.com
}

\begin{abstract}
With the rapid development of the Internet, the fan community communication presents new characteristics and effects. This paper takes the series 227 events of Zhan Xiao' s fan as an example and collect relevant data on Weibo for analysis through quantitative research method. The research shows that the characteristics of online fan community communication are multi-platform and multi-subject; fast speed, wide range and great influence; complexity, diversity and multiple layers. The communication effect focuses on the influence on idols themselves, fans groups, network subculture, network governance and other aspects. In view of this, some suggestions are put forward from four aspects, including idols' social responsibility, promotion of media literacy of fans and teenagers, respect for subculture, and strengthening of network governance order.
\end{abstract}

Keywords : Online fan community, Weibo, the series 227 events of Zhan Xiao's fan, characteristics of fan community communication, effects of fan community communication

\section{BACKGROUND}

Internet communication is a form of information exchange which uses the multimedia as the terminal, uses the optical fiber as the channel, and connects individuals and organizations together and can also interact with a "personalised" audience (Peng, 2009). In the Internet age, the way of dissemination of information have changed greatly and appeared the new characteristics. Firstly, the information updates faster in the Internet age, compared with the traditional way of spreading information. Secondly, the access to information has increased with the improvement of network technique. Thirdly, communication tends to be popular and civilian. As a result, people are no longer just the receivers of information, they are also the publishers of it. The freedom of speech of citizens shows a trend of enlargement. Last but not least, Internet communication has the feature of anonymity.

Some groups with unique hobbies, subcultures and their communities have developed quickly depending on the network platforms since those characteristics of Internet communication. For example,strangers, who have the same or similar hobbies, could gather on the Internet because of its connectivity, and form a virtual circle or a community of friends. As a result,this will generate new network communities and groups, which play important roles in reshaping cyber culture, fan culture, Two-dimensional culture and Tongren culture. The word Tongren(doujin) was introduced in Japanese, which means like-minded people. It is widely used to refer to a literary or artistic work in which fans recreate characters from a particular literary anime, film, or game that have nothing to do with the original, a generic term for fan fiction and fan art.

With the development of network groups, network communication of its virtuality and anonymity could also have negative impacts on society. For instance, some netizen or We-Media would spread false information on the Internet in order to grab the headlines,as a result, it will lead the wrong public opinions. What's worse,the irrational behaviors which are committed by fans sometimes may cause cyber violence. These misbehavior mentioned above will affect the network order and have an adverse impact on society to some extent. At the same time, these behaviors could also bring difficulties to state 
and social governance, especially for teenagers, so that it has caused the attention of the governments gradually.

Based on this, this paper wants to study how the fan groups collect and disseminate information in the network communities, and also the characteristics and effect of the dissemination. This study chooses the series 227 events of Zhan Xiao as an example. On the afternoon of February 24, 2020, a CP fan, which refers to a couple that fans are crazy about lovers in a variety show or a TV play, and always fantasize that they are also a couple in reality, of Zhan Xiao and Yibo Wang's published a Tongren novel called Xiazhui on AO3 website. The Tongren novel means that as long as the characters of the original works are borrowed, or even the novels written by the world outlook, they can basically be called Tongren novels.In this novel, the author feminizes the image of Zhan Xiao, who is now a famous male idol and star. Zhan Xiao's fans thought it was bad for their idol's on-screen image, so that they strongly resisted. Until February 26, 2020, some of Xiao's fans reported this novel and even the AO3 website. On the afternoon of February 26, these Xiao' fans began to report and attack other novels similar to Xiazhui and it went from reporting to calling the authorities. The author of Xiazhui was subjected to the human flesh search, and deleted the article. On the evening of February 26, 2020, some of Xiao's fans, especially loves Xiao only reported and attacked AO3 website, Lofter website, and other software under the slogan of "Protecting the environment for minors to access the Internet". At the same time, Xiao's fans were giving some apps a one-start ranting, including Baidu cloud, Douban, Zhihu etc. These things occupied the network public space, caused network violence and affected the majority of netizens, resulting in many netizens dissatisfied with Zhan Xiao and his fans. Because of the resisting of other netizen who don't like Zhan Xiao, some products endorsed by Zhan Xiao have been replaced by other stars' brand promotion, and the conflict between the two sides has become more intense. On February 29, AO3 website in mainland of China was blocked due to too many reports. On March 1, the head of fans posted a statement saying that the fans' behavior had nothing to do with the idol, and Zhan Xiao's studio also issued an apology at 9 PM that night.

The series 227 events of Zhan Xiao'fan resulted in a conflict between Xiao's CP fans, Xiao'anti fans, who focus on a star or an idol in order to darken and hate him or her instead of love, fans of Tongren culture and other netizens, which had attracted wide attention from the state, society, mainstream media, NPC deputies and other sectors. This paper will study the specific communication content, communication channels, communication characteristics of the fan communities in this event, and analyze the communication effect based on the data on Weibo.

\section{LITERATURE REVIEW}

Different scholars have different explanations of fans. In terms of external behavior, some scholars state that a fan is a group of people who spontaneously gather to adore or chase after a celebrity or a cultural phenomenon

(Shen \& Gao, 2020) . In addition, in terms of inner emotions, Yun Gao (2019) believes that fans are audiences for specific media content, and they are a kind of emotional union. Their difference from the general audience is mainly reflected in the emotional category -fans invest more in some aspects than others.

The word "fans" has been in the public view since 2005, when the Super Girl became the hottest phenomenon in the country. The birth of the Internet has profoundly changed the way of human production and life, and affected social relations and social structure, and it has also derived new social space and social practice (Xie, 2018). In the context of the development of the Internet, the meaning of fans has also changed. Nowadays, fans cover not only the field of film, TV series and songs, but also almost cover all fields related to cultural phenomena, characteristics, the way of expression and other aspects of it are more marked by the era, such as personality, craziness, innovation, transience, and even utility. As a result, fans have shaped a special kind of culture, and become the most loyal group of cultural consumers (Sun, 2006).

The Internet provides technique for the formation of fan groups and the exchange of information among members. The individual is the basis of a group. The group is made up of individuals who share a common goal and cooperate with each other. The communication and interaction mechanism between groups and members, and between members and members is called group communication (Guo, 2011). Sociologist Herbert Blumer symbolic interaction theory believes that in a group, members' mutual promotion and interaction reaction will stimulate another person, who will reflect according to the degree of stimulation, and infect and influence each other among group members to form group behavior (Zhang, 2015). Members of a fan community often exchange pictures and videos to spread information and share resources.

The Internet also strengthens the participation of fans, from the original one-way reception of information and passive participation in the dissemination of information to become the"publisher" of information.The Internet has increased the participation of fans, and also provided the possibility for fans to participate in the promotion of their idols, career planning and other activities. Zhang Qiang (2010) mentioned in The Power of Fans that today's fans are no longer a group of obscure and passive consumer media content under traditional mass media. The Internet liberates the unequal power relationship between media 
organizations and audiences, and the power of groups really breaks out.

In the past, fans were passive, and they could only follow the idols promoted by mass media, so that it was difficult for them to form their own independent judgments. After the emergence of Weibo, fans began to really participate in the construction of fan culture (Huang\&Song, 2016). According to Aiman Data \& Weibo: 2018 Fan White Paper (2019), as a representative social media where there are so many stars gathering, Weibo is also the largest fan gathering place in China. Weibo is the leader of the fan economy era, and the core witness of the fan economy.

Weibo fans, a new form of online fans, has developed in the 20th century under the tide of Internet technology. Therefore, in addition to the common characteristics of network fans, Weibo fans have generated new features. Fans-stars and fans-fans establish virtual social relations through micro blogs, which breaks down the barriers between fans and idols in the traditional media era to some extent (Li, 2012). Compared with the unicity of traditional media, Weibo integrates various media forms such as text, pictures and videos, which enriches the channels for fans to learn about their idols and promote the speed of obtaining information. At the same time, fans are free to choose the objects and contents. Moreover, through the communication advantage of Weibo, they can actively expressed their views and attitudes. The once obscure fan audience became the dominant communicator, transforming idolatry into a rich and complex participatory culture. Some scholars found that the foundation of fan culture in the Weibo era lies in the lack of social relationships in reality, and it forms a new kind of social relationship between stars and fans (Huang\&Song, 2016).

Some of the fans' voluntary public welfare behaviors can have a positive impact on the society. However, due to the low threshold and anonymity of network use, the cultural level of netizens is different, which requires the society to supervise and manage the network virtual society. In recent years, fans often fight for their idols' status, treatment and resources on the Internet, which not only affects the artists' own image and resources, but also has a negative effect on the social order, and may even cause network violence and other harms. Therefore, strengthening the governance of the network becomes a top priority. Some scholars pointed out that in the process of government promoting network content governance, the meaning of network content governance is gradually clear and the strategic height is constantly improved (Xie \& Zhu, 2020).

\section{RESEARCH CONTENT}

\section{1. research method and data collection}

In the process of studying the series 227 events of Zhan Xiao' $s$ fan, this paper has used the quantitative research method with the help of Weibo platform to extract relevant data, including Weibo contents related to Zhan Xiao and his studio, his fan club and its comments, the number of pageviews, forwarding and comments of top searches and other relevant important microblogs about the event. According to relevant statistics, @Fan Circle Aunt microblog data (2020) shows that in the first half of 2020, the most-searched topics related to Zhan Xiao (including Weibo, Tiktok and Headlines) reached 251, ranking first on the artist list. Through the data collection and analysis, the characteristics and effects of fan community communication are obtained.

\section{2. the features of fan club communication}

First of all, online fan club communication presents the characteristics of multi-platform and multi-subject. According to Aiman Data \& Weibo: 2018 Fan White Paper (2019), Weibo has developed into a major gathering place for fans, who spontaneously forming a "fan community". Fans can follow their favorite stars on Weibo, and join the relevant fan club and fan groups, in which they can support, comment and toward message on Weibo. Fans can even get the chance to interact with their idols. In addition, QQ, WeChat, Zhihu and Douban have also become platforms for fans to discuss, share and promote their idols. Compared with the traditional media era, fans receive information from their idols unilaterally from newspapers and TV. In the Internet era, fans are also spreaders and can recreate the content they are interested in.

Secondly, the fan community in the network era has the characteristics of fast propagation, wide propagation range and great influence. Fan groups usually use Weibo and Douban as platforms for content dissemination and bring about a series of influences. During the series 227 events of Zhan Xiao' s fan, his fans paid close attention to an article called Xiazhui immediately after it was published on Weibo. Opinion leaders in Xiao's fan community believed websites such as $\mathrm{AO} 3$ and Lofter were pornographic, and pointed out that these websites had a bad influence on teenagers. Therefore, under their leadership, Zhan Xiao' s fans quickly organized largescale reports on this article, AO3, Lofter and other websites. Their behavior was rapidly spread on the Weibo platform, which have attracted the attention and discussion of various media, fan groups and ordinary netizens. In addition, Zhiwei(2020), which is a data analysis platform of microblog, shows that multiple media participated in the report, including China Youth 
Net, China News Net, China Net and and other 5 mainstream media.

Zhiwei (2020) data shows that the event lasted for a total of 16 days and 11 hours on the Weibo platform, with a peak transmission speed of 1,487 per hour, which reflected the high attention of the media and netizens on the event,

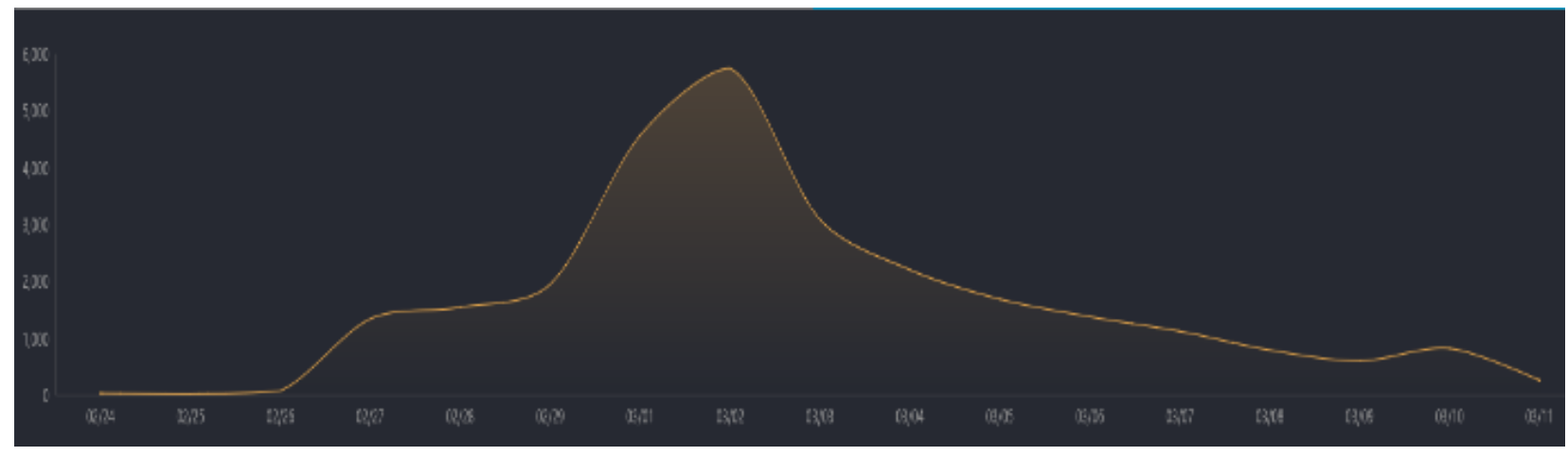

Picture1: propagation speed and propagation peak1 by date

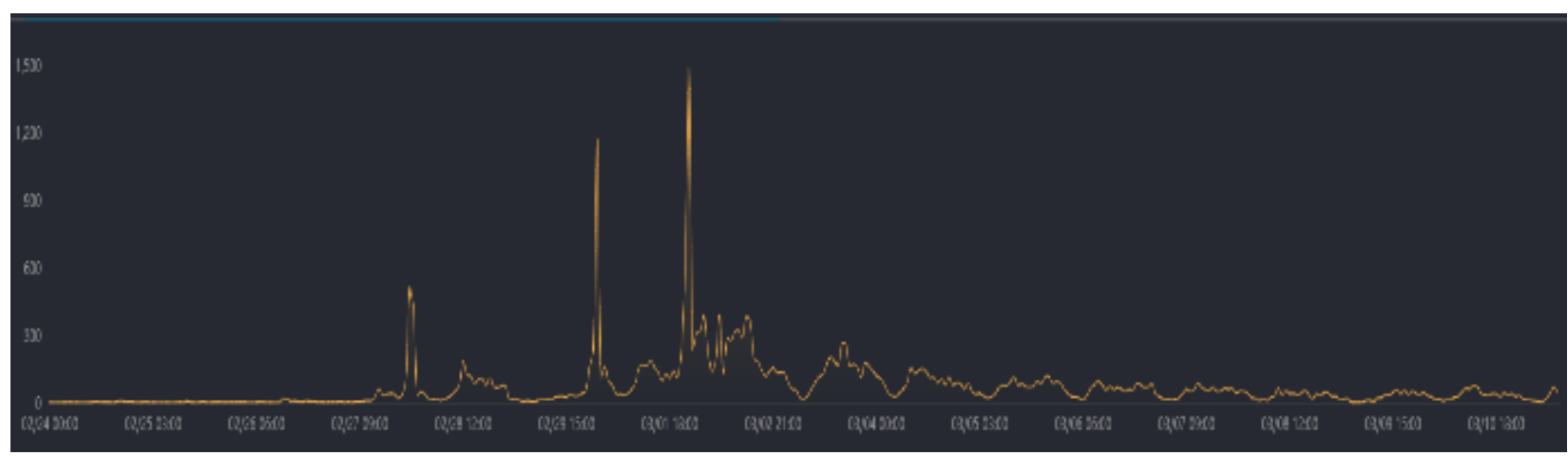

Picture2: propagation speed and propagation peak2 by hour

In addition, fan community communication has the characteristics of complexity, diversity and multiple circles. Fan community communication not only reflects the fan community communication among the internal members, but also reflected in the fan community and other communities or Internet users and other external members. Therefore, fan communities sometimes show the characteristics of multi-circle of communication. For example, when the series 227 events of Zhan Xiao' s fan broke out, his fans' behavior has also been questioned and attacked by CP fans, Xiao's anti fans, fans of Tongren culture and other netizens, causing a melee on the Internet. This shows that in the Internet age, Internet users are automatically divided into different camps due to different interests and hobbies, which may easily lead to the outbreak of online public opinion, online violence and the disorder of online order.

\section{3. fan club communication effect and network governance}

The series 227 events of Zhan Xiao's fan brings a lot of propagation effects. This paper analyzes the micro effect, macro effect, short term effect and long term effect. As for the micro effect, some netizens launched a "Anti Zhan Xiao" movement, believing that Zhan Xiao did not bear the corresponding social responsibility as a public figure. Apart from this, Xiao's star image and commercial value have been greatly affected. For example, some of Zhan Xiao's commercial endorsements were withdrawn and the scores of TV dramas decreased, which reflected that netizens have moral and social responsibility requirements for public figures and stars 。

As for the macro effect, from the perspective of youth subculture, AO3 and Lofter is a "pure land" of Tongren fans, and these websites are also the main platform for them to spread and exchange their Tongren culture. The blockade of AO3 is not conducive to the development of Tongren culture and even subculture in China.

As for the short term effect, Zhan Xiao's studio issued an apology on March 1, 2020, and Xiao's fan club and his fans also apologized on Weibo. According to the data, as of September 25, 2020, the apology statement on the event from Xiao's studio has been commented on more than 1 million times, transpond 386,000 times and received 12.25 million thumb up views (Zhan Xiao' studio, 2020). So far, the disorder caused by the public opinion storm on the Internet has come to an end.

As for the long term effect, the series 227 events of Zhan Xiao' s fan have aroused the government's 
attention to online fan club communication and youth group communication. On this basis, our country will develop the governance of network groups and network order. Mainstream media such as The Beijing News, Jiefang Daily and Supervisory Daily have also published articles to express their views on the incident. They all call for rational pursuit of stars and govern the behavior of fan clubs. What's more, these media also believe that artists should lead by example and give positive energy guidance to fans' behavior. On July 13, 2020, the relevant departments of the state have issued corresponding policies to guide teenagers' Internet use behavior and promote their healthy Internet access. Therefore, the series 227 events of Zhan Xiao's fan triggered the state to strengthen network supervision and management and standardize the network order.

\section{CONCLUSIONS}

By using quantitative research methods and taking the series 227 events of Zhan Xiao's fan as an example, this paper analyzed the characteristics and effects of fan community communication in the network era. The research showed that the online fan community communication had a lot of characteristics, such as multiplatform, multi-agent; fast speed, wide range and great influence; complexity, diversity and multi-circle. At the same time, it also brings great communication effect. It has a negative impact on Zhan Xiao himself and his business value, and is not conducive to the development and promotion of the Internet youth subculture. However, from the perspective of positive impact, the incident has promoted the country to strengthen the supervision and governance of the Internet, as well as regulate the online behavior of minors.

For idols, they need to actively take social responsibility and set an example in order to guide their fans' expressions and behaviors correctly. For the fan groups mainly composed of teenagers, they need to strengthen their media literacy, moral literacy and legal awareness, and surf the Internet properly. From the national level, the Chinese government needs to introduce relevant policies to guide young people to use the Internet correctly, build a network civilization, and prohibit such behaviors as abuse and online violence. From the cultural perspective, the fan groups also need to respect subculture and subculture groups, and develop subculture with the help of the Internet.

\section{REFERENCES}

[1] Peng, L. (2009). Introduction to Network Communication (second edition). China Renmin University Press.

[2] Lin, S \& G, Jing. (2020). Characteristics of Network Variety Shows from the Perspective of Fan Culture-
-Take Idol Producer and Produce 101 as examples. Youth Journalist, No.664(08), 79-80.

[3] Gao, Y.(2019).Research on fan Culture from the Perspective of Cross-cultural Communication. Youth Journalist, No.650(30), 32-33.

[4] Xie, XZ. (2018). The Connotation and Significance of Internet Thought. Journal of Peking University (Philosophy and Social Sciences Edition), 55(01), 117-123.

[5] Sun, HY. (2006). Ramble on the Phenomenon of Fans and its Cultural Interpretation. Modern Communication - Journal of Communication University of China(6), 7-9.

[6] Guo, QG. (2011). A Course in Communication (second edition). China Renmin University Press.

[7] Zhang, J.(2015). Research on Weibo User group Behavior Based on Complex Network. (Doctoral dissertation).

[8] Zhang, Q.(2010). Fans are Powerful. China Renmin University Press.

[9] [12] Huang, TT \& Song, QQ.(2016). Fan Culture Communication from the Perspective of Weibo. Editorial Friend, 000(010), 50-52,58.

[10] [15] Aiman Data \& Weibo: 2018 Fan White Paper, http://www.199it.com/archives/811475.html , December 23,2018.

[11] Li, Y. (2012). An Analysis of the Formation Mechanism of Weibo Fans. Press Outpost, 2011(001), 47-48.

[13] Xie, XZ \& Zhu, YY. (2020). Research on the Development Trend and Countermeasures of Network Content Governance. News and Writing(4).

[14] The most Searched List of Artists in the First Half of 2020, @ Fan Circle Aunt microblog data (2020), https://weibo.com/1746658980/J4XiWfPMX?type= comment\#_rnd1601431620741, June 3,2020.

[16] Zhan Xiao' s Fans Report AO3 and Other Websites Caused Hot Discussion Pieces Analysis,Zhiwei, https://ef.zhiweidata.com/app3/dist/analyse/519bd1 8c129d230510029761, March,2020.

[17] An apology, @ Zhan Xiao's studio, https://www.weibo.com/u/6643123988?is_all=1\#_1 oginLayer_1601379560714, September 25,2020 